\title{
PRODUTIVIDADE E QUALIDADE FISIOLÓGICA DE SEMENTES DE SOJA PROVENIENTES DE PLANTAS DESSECADAS COM O HERBICIDA CARFENTRAZONE-ETHYL
}

\author{
Gil M. de Sousa Câmara ${ }^{1}$ e Luciana A. Marques $^{2}$
}

\begin{abstract}
'Eng ${ }^{\circ}$. Agr ${ }^{\circ}$, M.Sc., Dr., Professor Associado. Departamento de Produção Vegetal, Escola Superior de Agricultura "Luiz de Queiroz", Universidade de São Paulo. Av. Pádua Dias, 11. Caixa Postal 9. Piracicaba, SP 13418-970 gmscamar@carpa.ciagri.usp.br

${ }^{2}$ Graduanda em Engenharia Agronômica, Estagiária em Iniciação Científica. Bolsista da FAPESP. Departamento de Produção Vegetal, Escola Superior de Agricultura "Luiz de Queiroz", Universidade de São Paulo. lamarque@ecarpa.ciagri.usp.br
\end{abstract}

\begin{abstract}
RESUMO
O experimento teve como objetivo avaliar o efeito do herbicida carfentrazone-ethyl aplicado como dessecante na cultura da soja, sobre a produtividade e a qualidade fisiológica das sementes. O delineamento experimental foi o de blocos ao acaso com dez tratamentos e quatro repetições e com parcela composta de 8 linhas, cada uma com $5 \mathrm{~m}$ de comprimento. Os tratamentos aplicados sobre o cultivar FT-Jatobá, foram os seguintes: 1) testemunha, sem dessecante; 2) carfentrazone-ethyl (50 g/ha); 3) carfentrazone-ethyl (70 g/ha); 4) carfentrazone-ethyl + MSMA (50 + $480 \mathrm{~g} / \mathrm{ha}) ; 5)$ carfentrazone-ethyl + MSMA (60 + $408 \mathrm{~g} / \mathrm{ha}) ; 6)$ carfentrazone-ethyl + MSMA (70 + $336 \mathrm{~g} / \mathrm{ha}) ; 7)$ MSMA (1200 g/ha); 8) amônio glufosinato $(500 \mathrm{~g} / \mathrm{ha}) ; 9)$ paraquat $(400 \mathrm{~g} / \mathrm{ha})$ e 10$)$ diuron/paraquat $(250 / 500 \mathrm{~g} / \mathrm{ha})$. Os produtos foram aplicados por meio de pulverizador costal pressurizado à base de $\mathrm{CO}_{2}$, entre os estádios fenológicos $\mathrm{R}_{6}$ e $\mathrm{R}_{7}$ e com umidade nos grãos entre 32 e $40 \%$. Foram avaliadas as seguintes características: produtividade de grãos, umidade de colheita, teste padrão de germinação, teste de primeira contagem, envelhecimento acelerado, umidade após envelhecimento acelerado, emergência em campo e condutividade elétrica. Concluiu-se que o herbicida carfentrazone-ethyl aplicado como dessecante na cultura da soja, individualmente nas doses de 50 e $70 \mathrm{~g} / \mathrm{ha}$ e em conjunto com o herbicida MSMA nas doses de 50+480, 60+408 e 70+336 g/ha, não interfere na produtividade e na qualidade fisiológica das sementes. Pelo fato de tratar-se de um novo herbicida, cuja eficiência como dessecante é pouco conhecida, recomenda-se a realização de mais pesquisas sobre o assunto.
\end{abstract}

Palavras-chave: dessecante, Glycine max, germinação, vigor, maturidade fisiológica.

\section{ABSTRACT \\ Productivity and physiological quality of soybean seed originated from plants desiccated by carfentrazone-ethyl}

The purpose of the experiment was to evaluate the application of carfentrazone herbicide as desiccant on a soybean crop and its effect on soybean seed yield and physiological quality. A randomized complete block design with 10 treatments and 4 replications was set up. The experimental plot consisted of 8 rows, each $5 \mathrm{~m}$ long. The following treatments were applied to plants of the FT-Jatobá cultivar: 1) control, without desiccant; 2) carfentrazone (50 g/ha); 3$)$ carfentrazone (70 g/ ha); 4) carfentrazone + MSMA $(50+480 \mathrm{~g} / \mathrm{ha}) ; 5)$ carfentrazone + MSMA $(60+408 \mathrm{~g} / \mathrm{ha}) ; 6)$ carfentrazone + MSMA (70 + $336 \mathrm{~g} / \mathrm{ha}) ; 7)$ MSMA (1200 g/ha); 8) amonium gluphosynato (500 g/ha); 9) paraquat (400 g/ha) and 10) diuron / paraquat $(250 / 500 \mathrm{~g} / \mathrm{ha})$. The desiccants were applied with a carbon dioxide-pressurized backpack sprayer, between $\mathrm{R}_{6}$ and $\mathrm{R}_{7}$ soybean development stages, the seeds having between 32 and 40\% humidity. The following characteristics were analyzed: 
water content, standard germination, first count of standard germination, accelerated aging, water content after accelerated aging, electric condutivity and seed yield. It was concluded that carfentrazone applied as a desiccant on soybean crops, individually in dosages of 50 and $70 \mathrm{~g} / \mathrm{ha}$ or with MSMA at dosages of $50+480,60+408$ and $70+336 \mathrm{~g} / \mathrm{ha}$, did not affect the soybean seed yield and their physiological quality. More studies on carfentrazone-ethyl as a desiccant of soybean is necessary.

Key words : desiccant, Glycine max, germination, vigor, physiological maturity.

\section{INTRODUÇÃO}

Atualmente a soja representa o agronegócio mais importante do Brasil, sendo a cultura líder em área cultivada, mercado consumidor de insumos agrícolas e maior captadora de divisas para o país, por meio da exportação de grãos, óleo e farelo.

Apesar da elevada produtividade de grãos encontrada em diferentes regiões produtoras, nem sempre ocorre o mesmo no que se refere à produção de sementes de elevada qualidade fisiológica e sanitária, principalmente nas áreas de expansão da cultura, acima do paralelo $24^{\circ} \mathrm{S}$, onde predominam temperaturas e precipitações mais acentuadas (Costa et al., 1983; Melfi, 1996). Em tais condições, a infestação tardia de mato, a ocorrência de percevejos sugadores de vagens, a deterioração das sementes nas vagens e o atraso de colheita são situações que podem prejudicar a produção de sementes (Andreoli \& Ebeltoft, 1979; Costa et al., 1983; Fraga,1988; Silva et al., 1999; Teófilo et al., 1999).

Com relação à colheita mecanizada, é importante que essa operação ocorra o mais próximo possível do ponto de maturidade fisiológica das sementes, quando o teor de umidade alcança níveis próximos de $25 \%$. Entretanto, esse alto teor de umidade, associado à grande quantidade de folhas e hastes ainda verdes, tornam impossível uma colheita mecânica eficaz (Costa et al., 1983).

Uma das maneiras de amenizar esse problema, seria a antecipação da maturação final da cultura em campo, por meio da aplicação de substâncias químicas denominadas genericamente de dessecantes, que promovem a aceleração da secagem dos grãos e também das folhas, haste e ramificações da soja (Andreoli \& Ebeltoft, 1979).

Além da secagem das sementes e das plantas, a dessecação das plantas daninhas proveniente da infestação tardia na área, a uniformidade da maturação, o melhor planejamento da colheita e a maior rapidez na operação de colheita, com menor índice de perdas, são outras vantagens apontadas para o uso de dessecantes na cultura da soja (Silva et al., 1999; Teófilo et al., 1999).

Andreoli \& Ebeltoft (1979) estudando os herbicidas glifosato e paraquat aplicados nas doses de 0,56 e $1,12 \mathrm{~kg} /$ ha, respectivamente, como dessecantes na cultura da soja, constataram que esses produtos não influenciaram na produtividade de soja e que a umidade das mesmas declinou muito rapidamente após o tratamento com dessecação, de maneira que os dessecantes não influenciaram a viabilidade das sementes.

Costa et al. (1983), em resultados de três anos de pesquisa com o uso dos dessecantes paraquat ( 2 l/ha) e bromoxinil (1 l/ha) em campos de produção de soja, cv. Davis, observaram uma queda acentuada na umidade das sementes, sendo que no intervalo de 4 a 5 dias a percentagem de umidade decresceu de 30 , para 18 a $17 \%$, ocorrendo antecipação de colheita de 4 a 9 dias.

Ratnayake \& Shaw (1992), estudando vários produtos dessecantes e épocas de aplicação, constataram que herbicidas aplicados no estádio $\mathrm{R}_{5}$ reduziram a produtividade da soja; nos estádios $\mathrm{R}_{5}$ e $\mathrm{R}_{6}$, paraquat reduziu a produtividade em 97 e $27 \%$, respectivamente; glufosinato reduziu em 96 e 31\%; glifosato reduziu em $88 \%$ em $\mathrm{R}_{5}$ e em $\mathrm{R}_{6}$, não afetou a produtividade da soja. Em $R_{7}$ e $R_{8}$, nenhum dos herbicidas reduziu a produtividade da soja. Quanto à germinação, somente glifosato reduziu-a, quando aplicado no estádio $R_{5}$, sendo observado também redução em $R_{6}$ e $R_{7}$. Paraquat e glufosinato não afetaram a germinação quando aplicados em qualquer dos estádios de desenvolvimento estudados.

Silva et al. (1999) constataram que as interações entre os efeitos dos dessecantes paraquat e paraquat + diquat $\mathrm{e}$ das épocas de aplicação na redução do teor de umidade da semente e da planta foram significativas, sendo que a secagem mostrou-se menos eficiente quando a dessecação foi realizada em plantas cujo grau de umidade era de $42 \%$. Assim, concluíram que além da colheita tradicional, as aplicações tardias também prejudicaram o vigor das sementes.

O avanço tecnológico voltado para a busca de elevadas produtividades e de maior eficiência nas operações agrícolas é um processo contínuo que resulta no desenvolvimento de novas técnicas e produtos. Carfentrazone-ethyl constitui-se em nova substância de ação herbicida, cujo uso como dessecante de culturas não é difundido, nem sua ação sobre a qualidade das sementes é conhecida, o que justificou a realização deste trabalho de pesquisa.

\section{MATERIAL E MÉTODOS}

O experimento foi conduzido no ano agrícola de 1999/ 2000, em área experimental da Escola Superior de Agricultura "Luiz de Queiroz", Universidade de São Paulo (ESALQ/ USP), localizada no município de Piracicaba, SP, 22 41' 30" 


\section{S, $47^{\circ} 38^{\prime} 30^{\prime \prime}$ O e a $546 \mathrm{~m}$ de altitude.}

O solo no local do ensaio é classificado como Podzólico Vermelho-Escuro Latossólico, com boa drenagem, textura muito argilosa, contendo $62 \%$ de argila total, $11 \%$ de silte e $27 \%$ de areia.

Como cultivar de soja utilizou-se o FT-Jatobá, semeado em 19/12/1999, de ciclo de maturação médio, de uso recomendado para o estado de São Paulo visando a produção de sementes.

O delineamento experimental foi o de blocos ao acaso com 10 tratamentos e 4 repetições, sendo as unidades experimentais constituídas de 8 linhas espaçadas a $0,45 \mathrm{~m}$ entre si e com $5 \mathrm{~m}$ de comprimento.

Como herbicidas de ação dessecante, foram utilizados os produtos descritos na Tabela 1, todos comparados a um único tratamento controle (testemunha) em que não houve a aplicação de dessecante.

As aplicações dos tratamentos ocorreram em 04/04/ 2000 , ocasião em que os plantas encontravam-se entre os estádios de desenvolvimento $\mathrm{R}_{6}$ e $\mathrm{R}_{7}$ e a umidade das sementes entre $32 \mathrm{e} 40 \%$. Para tal, foi utilizado pulverizador costal pressurizado à base de $\mathrm{CO}_{2}$, mantendo-se a pressão constante de $3,1 \mathrm{kgf} / \mathrm{cm}^{2}$ com vazão individual nos 4 bicos (Teejet TT 110015-VP) correspondente a 0,59 litros por minuto, aplicando-se volume de calda equivalente a 300 litros por hectare.As aplicações tiveram início às 11:30h e terminaram às $12: 33 \mathrm{~h}$, com a temperatura variando de 32 a $34^{\circ} \mathrm{e}$ a unidade relativa do ar entre 42 e $45 \%$.

A produtividade foi avaliada após a colheita das parcelas, por meio da pesagem da produção de três linhas úteis (4 m de comprimento) com correção dos valores a $13 \%$ de umidade, expressos em kg/ha. Para obtenção das sementes, o material colhido foi classificado em peneira 14 e beneficiado manualmente, descartando-se as sementes manchadas, deformadas e visivelmente doentes. Para avaliar a qualidade fisiológica das sementes, foram realizados os seguintes testes, padronizados pelas Regras para Análise de Sementes (Brasil, 1992): teste padrão de germinação, teste de primeira contagem da germinação e umidade. As características de vigor foram analisadas através dos testes de condutividade elétrica, envelhecimento acelerado e emergência em campo, descritos por Marcos Filho et al. (1987).

Os resultados foram submetidos à análise da variância, cujos valores do teste de $\mathrm{F}$ foram não significativos para todas as variáveis analisadas.

\section{RESULTADOS E DISCUSSÃO}

Nas condições de fotoperíodo do estado de São Paulo, o cultivar FT-Jatobá apresentou-se com eiclo de maturação um pouco mais longo, de maneira que os seus estádios fenológicos de máximo volume de grãos $(R)$ e de maturidade físiológica $(R)$ ocorreram no início do mêês de abril, que no ano de 2000 apresentou-se relativamente mais seco e mais quente. Dessa forma, a ação dessecante dos produtos utilizados, provavelmente foi favorecida por tais condições.

Três dias após a aplicação dos dessecantes, as plantas de todas as parcelas apresentaram-se extremamente dessecadas, com folhas, pecíolos e ramificações fortemente desidratadas. Aos 7 dias todas as parcelas se apresentavam em condições de colheita, inclusive aquelas correspondentes ao tratamento testemunha.

Nas Tabelas 2 a 5 encontram-se os resultados obtidos

Tabela 1. Herbicidas utilizados no experimento, nas diferentes doses. Piracicaba, SP. 2000.

\begin{tabular}{|c|c|c|c|c|c|}
\hline \multicolumn{2}{|c|}{ Herbicida } & \multirow{2}{*}{$\begin{array}{c}\text { Concentraçã } 0^{1} \\
(\mathrm{~g} / \mathrm{l})\end{array}$} & \multirow{2}{*}{ Formulação } & \multicolumn{2}{|c|}{ Dose } \\
\hline Nome Técnico & $\begin{array}{c}\text { Nome } \\
\text { Comercial }\end{array}$ & & & $\begin{array}{l}\text { i.a. } \\
(\mathrm{g} / \mathrm{h} a)\end{array}$ & $\begin{array}{l}\text { p.c. } \\
\text { (l/ha) }\end{array}$ \\
\hline Carfentrazone & Aurora & 400 & Concentrado emulsionável & $\begin{array}{l}50 \\
70\end{array}$ & $\begin{array}{l}0,125 \\
0,175\end{array}$ \\
\hline $\begin{array}{c}\text { Carfentrazone } \\
+ \\
\text { MSMA }\end{array}$ & $\begin{array}{c}\text { Aurora } \\
+ \\
\text { Daconate }\end{array}$ & $400+480$ & $\begin{array}{c}\text { Concentrado emulsionável } \\
+ \\
\text { Solução aquosa concentrada }\end{array}$ & $\begin{array}{l}50+480 \\
60+408 \\
70+336\end{array}$ & $\begin{array}{c}0,125+1,0 \\
0,150+0,85 \\
0,175+0,7\end{array}$ \\
\hline MSMA & Daconate & 480 & Solução aquosa concentrada & 1200 & 2,5 \\
\hline $\begin{array}{l}\text { Amônio } \\
\text { glufosinato }\end{array}$ & Finale & 200 & Concentrado solúvel & 500 & 2,5 \\
\hline Paraquat & Gramoxone & 200 & Solução aquosa concentrada & 400 & 2,0 \\
\hline Diuron/paraquat & Gramocil & $100 / 200$ & $\begin{array}{l}\text { Suspensão aquosa } \\
\text { concentrada }\end{array}$ & $250 / 500$ & 2,5 \\
\hline
\end{tabular}

${ }^{1}$ Concentração do princípio ativo (i.a.) no produto comercial (p.c.). 
Tabela 2. Valores observados para a umidade das sementes antes do início dos testes de laboratório e após o teste de envelhecimento acelerado (EA) e estádio fenológico médio (EFM) das plantas de soja por ocasião da aplicação dos tratamentos. Piracicaba, SP. 2000.

\begin{tabular}{|c|c|c|c|c|}
\hline Tratamento & $\begin{array}{c}\text { Dose } \\
(\mathrm{g} / \mathrm{h} \mathrm{a})\end{array}$ & $\begin{array}{l}\text { Umidade } \\
\text { Inicial } \\
(\%)\end{array}$ & EFM & $\begin{array}{c}\text { Umidade } \\
\text { Após E A } \\
(\%)\end{array}$ \\
\hline Controle & ---- & 9,1 & $\mathrm{R}_{6.8}$ & 25,5 \\
\hline Carfentrazone & 50 & 9,1 & $\mathrm{R}_{6.9}$ & 25,0 \\
\hline Carfentrazone & 70 & 7,2 & $\mathrm{R}_{6,7}$ & 25,3 \\
\hline Carfentrazone + MSMA & $50+480$ & 9,1 & $\mathrm{R}_{7}$ & 25,1 \\
\hline Carfentrazone + MSMA & $60+408$ & 9,1 & $\mathrm{R}_{7}$ & 25,3 \\
\hline Carfentrazone + MSMA & $70+336$ & 9,2 & $\mathrm{R}_{7}$ & 26,5 \\
\hline MSMA & 1200 & 9,3 & $\mathrm{R}_{6.9}$ & 25,1 \\
\hline Amônio glufosinato & 500 & 9,1 & $\mathbf{R}_{7}$ & 24,8 \\
\hline Paraquat & 400 & 8,5 & $\mathrm{R}_{7.1}$ & 24,9 \\
\hline Diuron/paraquat & $250 / 500$ & 8,9 & $\mathrm{R}_{6.9}$ & 25,2 \\
\hline C.V. $(\%)$ & & 7 & & 2,2 \\
\hline
\end{tabular}

Tabela 3. Valores observados para o teste padrão de germinação (TPG) e para o teste de emergência em campo (EC) de sementes de soja. Piracicaba, SP. 2000.

\begin{tabular}{lccccc}
\hline \multicolumn{1}{c}{ Tratamento } & $\begin{array}{c}\text { Dose } \\
(\mathbf{g} / \mathbf{h a})\end{array}$ & $\begin{array}{c}\text { TPG } \\
(\mathbf{\%})\end{array}$ & $\begin{array}{c}\mathbf{T P G}^{2} \\
\mathbf{( \% )}\end{array}$ & $\begin{array}{c}\mathbf{E C} \mathbf{1}^{\mathbf{1}} \\
\mathbf{( \% )}\end{array}$ & $\begin{array}{c}\mathbf{E C}^{2} \\
\mathbf{( \% )}\end{array}$ \\
\hline Controle & - & 87,9 & 69,6 & 87,7 & 69,4 \\
Carfentrazone-ethyl & 50 & 89,0 & 70,6 & 88,6 & 70,2 \\
Carfentrazone-ethyl & 70 & 84,1 & 66,5 & 92,3 & 74,0 \\
Carfentrazone-ethyl+MSMA & $50+480$ & 97,0 & 80,0 & 93,8 & 75,6 \\
Carfentrazone-ethyl+MSMA & $60+408$ & 93,2 & 74,8 & 92,0 & 73,6 \\
Carfentrazone-ethyl+MSMA & $70+336$ & 91,0 & 72,4 & 87,4 & 69,2 \\
MSMA & 1200 & 94,1 & 76,0 & 94,2 & 76,1 \\
Amônio glufosinato & 500 & 87,4 & 69,2 & 90,4 & 72,0 \\
Paraquat & 400 & 92,6 & 74,2 & 93,0 & 74,7 \\
Diuron/paraquat & $250 / 500$ & 93,1 & 74,8 & 90,2 & 71,7 \\
\hline C.V. (\%) & & & 9,6 & & 11,5 \\
Teste F & & & $\mathrm{NS}$ & & $\mathrm{NS}$ \\
\hline
\end{tabular}

${ }^{1}$ Dados originais.

${ }^{2}$ Dados transformados segundo a expressão arc seno de $(\mathrm{x} / 100)^{0.5}$. 
Produtividade e qualidade fisiológica...

Tabela 4. Valores observados para o teste de primeira contagem (PC) e para o teste de envelhecimento acelerado (EA) de sementes de soja. Piracicaba, SP. 2000.

\begin{tabular}{lccccc}
\hline \multicolumn{1}{c}{ Tratamento } & $\begin{array}{c}\text { Dose } \\
(\mathbf{g} / \mathbf{h a})\end{array}$ & $\begin{array}{c}\mathbf{P C}^{\mathbf{1}} \\
(\mathbf{\%})\end{array}$ & $\begin{array}{c}\mathbf{P C}^{\mathbf{2}} \\
\mathbf{( \% )}\end{array}$ & $\begin{array}{c}\mathbf{E A}^{\mathbf{1}} \\
\mathbf{( \% )}\end{array}$ & $\begin{array}{c}\mathbf{E A}^{\mathbf{2}} \\
\mathbf{( \% )}\end{array}$ \\
\hline Controle & - & 84,2 & 66,6 & 79,3 & 63,3 \\
Carfentrazone-ethyl & 50 & 87,1 & 69,0 & 84,3 & 67,2 \\
Carfentrazone-ethyl & 70 & 85,4 & 67,5 & 77,5 & 63,3 \\
Carfentrazone-ethyl+MSMA & $50+480$ & 94,7 & 76,8 & 88,0 & 70,0 \\
Carfentrazone-ethyl+MSMA & $60+408$ & 92,0 & 73,5 & 90,3 & 72,5 \\
Carfentrazone-ethyl+MSMA & $70+336$ & 90,7 & 72,3 & 81,8 & 65,9 \\
MSMA & 1200 & 93,6 & 75,3 & 90,3 & 72,3 \\
Amônio glufosinato & 500 & 75,5 & 60,4 & 75,0 & 60,3 \\
Paraquat & 400 & 90,8 & 72,4 & 92,5 & 74,3 \\
Diuron/paraquat & $250 / 500$ & 91,6 & 73,2 & 87,3 & 69,3 \\
\hline C.V. (\%) & & & 10,3 & & 10,9 \\
Teste F & & & NS & & NS \\
\hline
\end{tabular}

'Dados originais

${ }^{2}$ Dados transformados segundo a expressão arc seno de $(\mathrm{x} / 100)^{0,5}$

Tabela 5. Valores observados para o teste de condutividade elétrica (CE) e para a produtividade de grãos de soja. Piracicaba, SP. 2000.

\begin{tabular}{lccc}
\hline \multicolumn{1}{c}{ Tratamento } & $\begin{array}{c}\text { Dose } \\
(\mathbf{g} / \mathbf{h a})\end{array}$ & $\begin{array}{c}\mathbf{C E} \\
(\boldsymbol{\mu} \mathbf{S} / \mathbf{g} / \mathbf{c m})\end{array}$ & $\begin{array}{c}\text { Rendimento } \\
(\mathbf{k g} / \mathbf{h a})\end{array}$ \\
\hline Controle & - & 52,9 & 1501,7 \\
Carfentrazone-ethyl & 50 & 52,5 & 1593,9 \\
Carfentrazone-ethyl & 70 & 56,2 & 1625,3 \\
Carfentrazone-ethyl+MSMA & $50+480$ & 48,8 & 1555,2 \\
Carfentrazone-ethyl+MSMA & $60+408$ & 53,0 & 1825,4 \\
Carfentrazone-ethyl+MSMA & $70+336$ & 53,6 & 1478,9 \\
MSMA & 1200 & 46,1 & 1482,1 \\
Amônio glufosinato & 500 & 52,6 & 1307,6 \\
Paraquat & 400 & 47,2 & 1768,1 \\
Diuron/paraquat & $250 / 500$ & 48,7 & 1730,5 \\
\hline C.V. (\%) & & 14,1 & 15,3 \\
Teste F & & $\mathrm{NS}$ & $\mathrm{NS}$ \\
\hline
\end{tabular}


para todas as variáveis analisadas estatisticamente. Nenhuma das características avaliadas sofreu influência dos tratamentos aplicados, indicando que o uso do herbicida carfentrazone-ethyl como dessecante da cultura da soja não interfere na qualidade fisiológica das sementes e nem na produtividade de grãos. Entretanto, as condições de temperatura e umidade por ocasião da aplicação dos tratamentos, associada ao ciclo mais longo do cultivar FT-Jatobá e a umidade das sementes, menor que $40 \%$ nos estádios $\mathrm{R}_{6}$ e $\mathrm{R}_{7}$, provavelmente tenham contribuído para a maior eficiência dos produtos como agentes dessecantes.

\section{CONCLUSÕES}

O herbicida carfentrazone-ethyl aplicado como dessecante nas doses de 50 e $70 \mathrm{~g} / \mathrm{ha}$ e em conjunto com o herbicida MSMA nas doses de 50+480, 60+408 e 70+336 $\mathrm{g} / \mathrm{ha}$, não interfere na qualidade fisiológica das sementes e nem na produtividade de grãos da cultura da soja, cultivar FT-Jatobá.

Pelo fato de tratar-se de um novo herbicida, cuja eficiência como dessecante é pouco conhecida, recomenda-se à realização de mais pesquisas sobre o assunto.

\section{LITERATURA CITADA}

ANDREOLI, C.; EBELTOFT, D. C. Dessecantes no rendimento e na qualidade da semente de soja. Pesquisa Agropecuária Brasileira, v. 14, n. 2, p. 135-139, 1979.

BRASIL. Ministério da Agricultura e Reforma Agrária. Regras para análise de sementes. Brasília: DNDV/ CLAV, 1992.365p.

COSTA, N. P.; FRANÇA NETO, J. B.; PEREIRA, L. A. G.; HENNING, A. A.; TURKIEWICK, L.; DIAS, M. C. Antecipação da colheita de sementes de soja através do uso de dessecantes. Revista Brasileira de Sementes, v. 5, n. 3, p.183-198, 1983.

FRAGA, A. C. Estudo sobre a utilização de dessecantes na produção de sementes de soja. Viçosa, 1988. $91 \mathrm{p}$. Tese (Doutorado). Universidade Federal de Viçosa.

MARCOS FILHO, J; CÍCERO, S. M.; SILVA. W. R. Avaliação da qualidade das sementes. Piracicaba, SP: FEALQ, 1987. 230 p.

MELFI, F. Efeito da dessecação química sobre a qualidade de sementes das variedades de soja Doko e Savana. Viçosa, 1996. 64p. Tese (Mestrado). Universidade Federal de Viçosa.

RATNAYAKE, S.; SHAW, D. R. Effects of harvest-aid herbicides on soybean (Glycine $\max$ ) seed yield and quality. Weed Technology, v. 6 : 339-344, 1992.

Silva, A. A.; DOMINGOS, M.; CARDOSO, A. A. Efeito do Paraquat e da mistura Paraquat + Diquat, como dessecantes, aplicados em diferentes épocas, no rendimento e na qualidade fisiológica das sementes de feijão. Revista Ceres, v. 46, n. 265, p. 230-250, 1999.

TEÓFILO, E. M.; MEDEIROS FILHO, S.; ANDRADE, M. J. B. Ação de dessecantes sobre o feijoeiro no inverno primavera. Revista Ceres, v. 46, n. 267, p. 471-492, 1999.

\section{AGRADECIMENTOS}

À Engenheira Agrônoma Helena Maria Chamma, responsável técnica pelo Laboratório de Análise de Sementes do Departamento de Produção Vegetal da ESALQ/USP, pelo apoio e orientação na rotina de análises e de testes de vigor das sementes de soja. 